\title{
Association of Waugh Syndrome with Intestinal Volvulus in an Infant
}

Ivonete Siviero ${ }^{1}$, Ivens Baker Méio ${ }^{1}$, Danielle Nunes Forny ${ }^{2}$, Marcos Antonio Turcatel², André Lima da Cunha $^{2}$, Betina Carla Bertrand Simões ${ }^{2}$

${ }^{1}$ Division of Pediatric Surgery, Department of Surgery, Medical School of Federal University of Rio de Janeiro, Rio de Janeiro, Brazil; ${ }^{2}$ Division of Pediatric Surgery, Instituto de Pediatria e Puericultura Martagão Gesteira, Federal University of Rio de Janeiro, Rio de Janeiro, Brazil.

\section{Corresponding Author:}

Dr. Ivonete Siviero

Email: ivonetesiviero@ufrj.br

This is an Open Access article distributed under the terms of the Creative Commons Attribution License (creativecommons.org/ licenses/by/3.0).

Received Accepted

Published

October 14, 2016

March 28, 2017

April 20, 2017

\begin{abstract}
Background: The association of intestinal malrotation and intussusception is known as Waugh's syndrome. We report a case of a 3-month-old infant with this syndrome and volvulus of the intussuscepted intestinal segment. Case Report: A three day old infant with failure to thrive presented with acute intestinal hemorrhage. Tubular mass on abdomen examination, gasless rectal ampulla on X-ray, and intussusception on ultrasound was suggestive of intestinal obstruction. Laparotomy showed ileo-colic intussusception, volvulus of intussuscepted segment, and intestinal malrotation. Conclusion: We emphasize the early diagnosis and urgent operative treatment to avoid vascular compromise of the midgut.
\end{abstract}

Keywords: Infant, Intestinal Volvulus, Intussusception, Syndrome.

\section{Introduction}

Intussusception and intestinal malrotation are common causes of intestinal obstruction in infants and children. However, the simultaneous occurrence of intestinal invagination and malrotation (Waugh's syndrome), and bowel volvulus is very rare [1]. Successful outcome of this clinical condition depends on prompt diagnosis and timely surgical intervention, to avoid serious complications such as vascular compromise of the gut due to associated volvulus. Delay in diagnosis can lead to an increase in mortality and morbidity. We report association of Waugh syndrome and volvulus of the intussuscepted intestinal segment in a 3 month old infant and we emphasize the need for urgent diagnosis and surgical treatment of this rare cause of intestinal obstruction.

\section{Case Report}

A three-month old female infant in pediatric ambulatory care for failure to thrive, presented at the emergency room with acute intestinal hemorrhage, excessive crying, an episode of yellowish vomit, and food refusal, which evolved over a period of 6 hours. The mother denied episodes of fever. On clinical assessment the infant was undernourished, pale, dehydrated and lethargic. Abdominal examination revealed mild distension and a tubular mass was palpable in the left lower quadrant. On rectal examination a large amount of blood was released and a mass could be palpated above the rectum. The blood count showed anemia. Serum electrolytes and blood gases had no abnormalities. A plain X-ray film of abdomen showed a poor intestinal gas distribution and a gasless rectal ampulla [Fig.1]. Ultrasonography confirmed the hypothesis of intussusception. Intravenous fluids were administrated before surgery. During laparotomy an ileo-colic intussusception, which had progressed into sigmoid colon, volvulus of intussuscepted segment, and intestinal malrotation were found. After volvulus reduction, Ladd's procedure was performed, allowing the 
intussusception to be manually reduced. As there were no signs of gangrene, there was no need for intestinal resection. Patient evolution was uneventful, and the infant was discharged on the $5^{\text {th }}$ day post-surgery. During one year of outpatient follow-up, weight and height gain were normal for her age.

\section{Discussion}

Idiopathic intussusception is a frequent cause of intestinal obstruction in infants. Its clinical manifestations are cramping abdominal pain, vomiting, abdominal distension and bloody mucous stools ("currant jelly"). In most cases the intussusception is ileo-colic and a pattern of small bowel distension can be seen in plain film of abdomen [2].

Intussusception and intestinal malrotation is a rare association, named Waugh's syndrome by Brereton et al [1]. These authors described, in a prospective study, a high incidence of malrotation in patients with intussusception. However, fewer than 60 cases described to date in the English literature [3]. Breckon and Hardley suggested that abnormal fixation and mobility of the mesentery of the ileum and ascending colon are important factors in the etiology of intussusception in Waugh's syndrome [4].

There are reports of intussusception following Ladd's procedure, suggesting that this procedure facilitates intussusception after surgery due to caecum mobility [5]. The simultaneous occurrence of intestinal malrotation, midgut volvulus and intussusception is even more unusual, with only fourcases reported in literature [6-9]. These four patients presented with abdominal distension, two of them with small bowel distension [6,7], one with gastric distension and a gasless intestine in the plain film of abdomen [8] and in a patient a plain abdominal radiograph was not done [9]. Inan et al. suggested that the absence of abdominal distension and paucity of gas distally on plain films may be two possible indicators of Waugh's syndrome

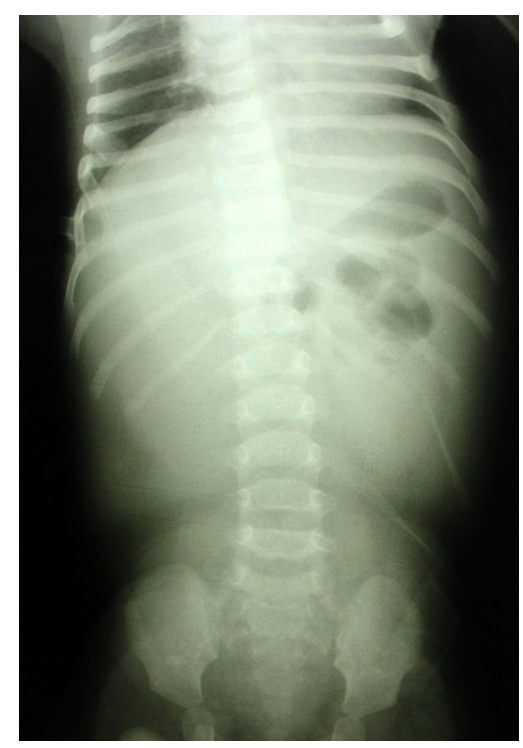

Fig.1: Plain abdominal film showing a gasless intestine.

[10]. We suggest that intestinal gas poverty in the presence of abdominal distension in an infant with ultrasonography finding of intestinal invagination, as was noticed in this case, may be considered an indicator of Waugh's syndrome with intestinal volvulus.

Hydrostatic or pneumatic reduction has been widely and successfully used to treat idiopathic intussusception [2]. In Waugh's syndrome, reduction is more difficult, but this procedure may unveil the cecal malposition, suggesting midgut malrotation $[11,12]$. However, if volvulus is suspected, surgical treatment should be performed immediately to avoid vascular compromise of the gut. In the present case, there was a large intestinal bleeding that indicated intestinal suffering and urgent surgical treatment. The malrotation diagnosis was only made during the surgery. High degree of suspicion and knowledge of this association is required to avoid the damages of a late diagnosis and treatment.

\section{Conclusion}

In infants with a history of failure to thrive, a clinical presentation suggestive of intussusception, poor feeding and change in the pattern of gas 
distribution on plain abdominal film, the possibility of malrotation and volvulus should be ruled out. Intestinal gas poverty in the presence of abdominal distension in an infant with ultrasonography suggestive of intestinal invagination may indicate this diagnosis. Pediatricians, surgeons and radiologists should be aware of this association to avoid an abdominal catastrophe caused by intestinal necrosis due to a delayed diagnosis.

Contributors: IS: supervised the diagnosis, management of the case and revised the manuscript; IBM: searched the literature and drafted the manuscript; DNF, MAT: searched the literature and critically reviewed the manuscript. ALC, BCBS: searched the literature and critical inputs into manuscript. IS will act as guarantor. All authors approved the final version of the manuscript.

Funding: None; Competing interests: None stated.

\section{References}

1. Brereton RJ, Taylor B, Hall CM. Intussusception and intestinal malrotation in infants: Waugh' syndrome. Br J Surg. 1986;73:55-57.

2. Lopes J, Huddart SN. Intussusception. Surgery. 2013;31:626-630.

3. Oliveira RCF, Paolilo, R, Fernandes JC, Fernandes
I, Bousso A. Case report - Child Waugh's syndrome. Pediatr Crit Care Med. 2014;15:158.

4. Breckon VM, Hadley GP. Waugh's syndrome: a report of six patients. Pediatr Surg Int. 2000;16:370-373.

5. Kidd J, Jackson R, Wagner CW, Smith SD. Intussusception following the Ladd procedure. Arch Surg. 2000;135:713-715.

6. Ornstein MH, Lund RJ. Simultaneous occurrence of malrotation volvulus and intussusception in an infant. $\mathrm{Br}$ J Surg. 1981;68:440-441.

7. Burke TE, Fitzgerald RJ. Intussusception, volvulus and malrotation. Aust N S J Surg. 1985;55:73-74.

8. Jain SK. Intestinal malrotation and midgut volvulus with intussusception: an unusual association. Arch Surg. 1989; $124: 509$.

9. Lukong CS, Maitama MI, Aminu B, Mshelbwala PM, Ameh EA. Intestinal malrotation and midgut volvulus coexisting with intussusception. South African J Surg. 2007;45:30.

10. Inan M, Basaran UN, Ayvaz S, Pul M. Waugh's Syndrome: Report of Two Cases. J Pediatr Surg. 2004;39:110-111.

11. Lobo E, Daneman A, Fields JM, Keller MS, Alton DJ, Shandling B. The diagnosis of malrotation during air enema procedure. Pediatr Radiol. 1997;27:606-608.

12. Luo $\mathrm{CC}$, Wang $\mathrm{CR}$, Chiu $\mathrm{CH}$. Intussusception and intestinal malrotation in an infant: a case report. Pediatr Surg Int. 2003;19:413-414. 